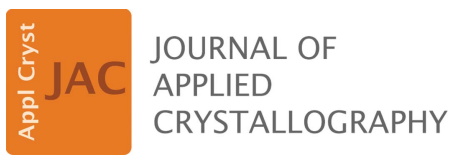

ISSN 1600-5767
Keywords: book reviews; neutron scattering; condensed matter

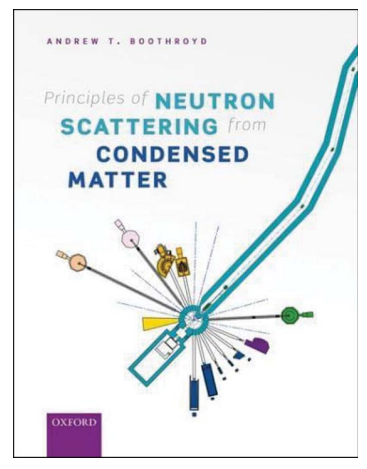

\section{Principles of Neutron Scattering from Condensed Matter. By Andrew T. Boothroyd. Oxford University Press, 2020. Pp. 512. Price GBP 55.00. ISBN 9780198862314.}

\author{
Thomas Proffen*
}

Oak Ridge National Laboratory, PO Box 2008, Oak Ridge, TN 37831, USA. *Correspondence e-mail: tproffen@ornl.gov

Neutron scattering user facilities are increasingly used by 'non-expert' users, opening neutron scattering to new science fields and greatly increasing the scientific impact of these facilities. This book aims to bring the needed introduction to the expanding area of neutron scattering techniques to this wider audience of scientists using neutron scattering as part of their experimental portfolio. In my view, it completely succeeds in that goal and provides the right balance of introduction and in-depth discussion. I also like the layout of the book, with some figures and notes in the margin making it easy to read and navigate. As a 'long-time' neutron scatterer, I am excited to add this book to my book shelf.

The first chapter discusses basic concepts from properties of the neutron to neutron sources and interactions with matter. This chapter also includes a discussion of cross sections and the concept of coherent versus incoherent neutron scattering. Chapter 2 deals with diffraction within the static approximation. The reader is introduced to diffraction from crystals, disordered crystalline materials, liquids, disordered solids and molecular systems. The chapter concludes with an introduction to small-angle neutron scattering.

Chapters 3 and 4 go more into fundamentals and the foundations of neutron scattering, covering, respectively, the kinematical theory of scattering and the interaction potential of neutrons with matter. In Chapter 5, the author describes nuclear scattering. After introducing the harmonic oscillator to illustrate some basic concepts, the chapter covers crystalline solids, correlation functions, spin rules, dynamics of disordered and complex systems, quasi-elastic scattering, and neutron spin echo spectroscopy. I like the way the chapter is structured and introduces the different concepts neutron users will come across as they measure and analyse neutron scattering data. One of the great strengths of neutron scattering is the ability to probe magnetism, and Chapter 6 covers the basics and general properties of magnetic scattering, introducing the magnetic cross section, the dipole approximation and magnetic form factors. Following this introduction, Chapter 7 dives into magnetic diffraction, including polarized neutron diffraction and magnetic diffuse scattering. Magnetic excitations are covered in Chapter 8, concluding the discussion of neutron scattering measurements of magnetic materials. Chapter 9 is titled Neutron Optics and covers dynamical theory, neutron reflectivity, neutron imaging and a discussion of extinction. Personally, I am not sure this grouping of topics in Chapter 9 is as intuitive as the other chapters in the book. The final chapter is dedicated to discussing practical aspects of neutron scattering and provides an excellent overview of aspects researchers need to consider when planning experiments and measuring or analysing neutron scattering data. I love that the author devoted a separate chapter to these topics rather than having the reader hunt for this information across an entire textbook as is the case with many other books. The book ends with an extensive appendix providing detailed information on mathematical definitions, quantum theory and linear response theory.

In my view this is an excellent book discussing the principles of neutron scattering from condensed matter. It is accessible to researchers new to neutron scattering and provides sufficient detail to become the go-to textbook for experienced neutron scatterers in the field. In fact, in addition to reading it for writing this review, I have already used it to look things up for my own research. 